
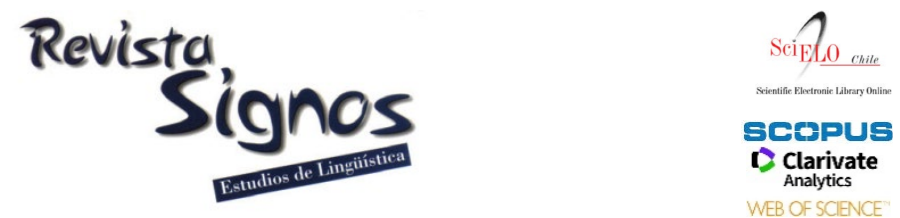

\title{
Introducción a la sección monográfica: Alteraciones y deterioro de la competencia lingüística en la Enfermedad de Alzheimer
}

La Enfermedad de Alzheimer (EA), la demencia más común de nuestros tiempos, es una patología neurodegenerativa con una innegable afectación lingüística. No obstante, a pesar de que el propio Alois Alzheimer refería que el mal que posteriormente se conocería por su nombre implicaba cambios sustanciales en el lenguaje de los afectados, el estudio de los mismos no ha recibido atención adecuada en la investigación propiamente lingüística, relegando la tarea a los ámbitos de estudio como la psicología o la neurociencia. Actualmente, los especialistas en demencias no dudan de que las alteraciones del lenguaje constituyan uno de los primeros síntomas de EA y que merecen una descripción pormenorizada y especializada dado su gran potencial no solo para la detección de la enfermedad de forma temprana, sino también para el aporte de evidencias a la teoría lingüística y a la comprensión científica del origen, funcionamiento y pérdida del lenguaje en los humanos.

Esta sección monográfica de Revista Signos. Estudios de Lingüística es uno de los primeros intentos -el primero en el ámbito hispanohablante- que pretende ofrecer un panorama sobre las afectaciones del lenguaje más destacables de personas con EA desde una perspectiva lingüística. Reúne para ello seis investigaciones a cargo de reconocidos especialistas en el estudio del lenguaje en demencia que, basándose en teorías y métodos propios de la lingüística experimental, afrontan los diferentes aspectos del deterioro de la capacidad cognitiva más destacable del ser humano bajo efecto de procesos neurodegenerativos. En este sentido, las aportaciones de los seis artículos que componen esta sección monográfica giran en torno a tres ejes temáticos: la habilidad lingüística en la demencia; las alteraciones discursivas derivadas de la neurodegeneración; y la afectación léxico-semántica en EA.

Los dos artículos del primer bloque se focalizan en la definición y la descripción de las alteraciones que sufren las personas con demencia a nivel de su habilidad y competencia lingüística en general. El trabajo a cargo de María Cruz Pérez Lancho y Sergio García Bercianos, titulado Caracterización del lenguaje en las variantes lingüsticas de la 
Afasia Progresiva Primaria, ofrece una versión panorámica de las propiedades de diferentes subtipos de este conjunto de trastornos lingüísticos por deterioro neurocognitivo, acercando al lector a un estado de cuestión actualizado y sistemático sobre los rasgos del lenguaje esperables en una demencia. A continuación, Mónica Salazar Villanea, en su artículo Narrativa autobiográfica y reminiscencias en costarricenses con Alzheimer, profundiza en cómo las narrativas autobiográficas de las personas con demencia pueden dar cuenta de tal afectación lingüística global y escalonada, que muestra la anterioridad de las alteraciones del discurso a las desviaciones de corte fundamentalmente semántico.

En el segundo bloque de la sección, el lector podrá darse cuenta de la especificidad de las alteraciones del discurso en demencia a partir de dos trabajos centrados en las propiedades discursivas par excellence: la coherencia y el lenguaje figurado. Estamos, sin duda alguna, ante dos aportaciones de gran relevancia científica, ya que el estudio del discurso en EA es un ámbito de investigación manifiestamente reciente y novedoso. La contribución de Anayeli Paulino, Gerardo Sierra, Iria da Cunha y Laura Hernández-Domínguez, titulada El análisis de las relaciones discursivas como recurso en el seguimiento de la Enfermedad de Alzheimer se basa en la Teoría de la Estructura Retórica para determinar cómo quedan afectadas las relaciones retóricas subyacentes a la conversación en las personas con demencia frente a las personas mayores sanas. Por su parte, Ana Varela Suárez, en El empleo del lenguaje figurado en el discurso de personas con demencia, analiza la afectación cualitativa y cuantitativa de los diferentes recursos figurados en la expresión oral de las personas con demencia, indagando en su funcionalidad y su valor discursivos.

El tercero, y el último bloque de artículos se centra en el aspecto más estudiado de la afectación lingüística en la Enfermedad de Alzheimer -la léxico-semántica-, pero no por ello tiene menos valor, pues ofrece una perspectiva renovada sobre qué ocurre con las palabras y los conceptos con el avance de la neurodegeneración. En Relaciones semánticas en adultos mayores sanos, Deterioro Cognitivo Leve y Enfermedad de Alzheimer, Lina Grasso y Gastón Saux se centran en las propiedades de la afectación semántica en la demencia, estudiando los factores y los atributos categoriales más deteriorados por los procesos neurodegenerativos. Por su parte, Olga Ivanova, Juan José García Meilán, Israel Martínez-Nicolás y Thide E. Llorente, en La habilidad léxico-semántica en la Enfermedad de Alzheimer: un estudio de la fluidez verbal con categorías semánticas, indagan en qué categorías semánticas son más afectadas por las alteraciones cognitivas en la demencia y qué cambios metodológicos conlleva en sí la observación de la diferenciación del deterioro semántico discontinuo.

Con los seis trabajos que la integran, esta sección monográfica hace una contribución esencial, novedosa y necesaria al estado de la cuestión sobre las alteraciones lingüísticas en la Enfermedad de Alzheimer. En su gestación y preparación, fue esencial el papel desempeñado por el Prof. Dr. Giovanni Parodi, 
Editor en Jefe de la Revista Signos. Estudios de Lingüística, quien aceptó, apoyó y veló durante todo el proceso porque este proyecto saliera adelante con éxito. Nuestros más sinceros agradecimientos tanto a él, como a todos aquellos investigadores que han participado en la revisión anónima de los trabajos y, con ello, han contribuido de forma sustancial a la calidad del material que a continuación les presentamos.

\section{Dra. Olga Ivanova}

\section{Dr. Juan José García Meilán}

Editores Invitados

25 de septiembre, 2019 